\title{
Prospective evaluation of cardiovascular-risk surrogates via lifestyle and adherence to the Mediterranean diet: Results from PERSEAS/The Elafonisos-Area Study
}

\author{
Christina Voulgari $^{1 *}$, Ioannis Kyriazis ${ }^{2}$, Ioannis Ioannidis ${ }^{3}$, Charilaos Dimosthenopoulos ${ }^{4}$, Erifili Hatziagelaki ${ }^{5}$ and Hellenic Medical Society \\ for the study of Risk Factors in Vascular Diseases-The Perseas Study Group \\ ${ }^{1}$ Fifth Department of Internal Medicine and Infectious Disease Unit, "Evaggelismos” Athens General Hospital, Greece \\ ${ }^{2}$ Department of Internal Medicine and Diabetes Outpatient Clinic, "KAT" Attica General Hospital, Greece \\ ${ }^{3}$ First Department of Internal Medicine and Diabetes Outpatient Clinic, "Konstantopouleio" General Hospital of Nea Ionia and Patision, Greece \\ ${ }^{4}$ Department of Clinical Nutrition, National and Kapodistrian University of Athens, "Laiko" General Hospital, Greece \\ ${ }^{5}$ Second Department of Internal Medicine, Research Institute and Diabetes Center, National and Kapodistrian University of Athens, Athens Medical School, \\ "Attikon" University Hospital, Greece
}

\begin{abstract}
Background: Lifestyle and nutrition are main causes of obesity, and cardiovascular disease. PERSEAS (Prospective Evaluation of vascular-Risk Surrogates: the Elafonisos-Area Study) is an epidemiological prospective study aiming to assess the physical course of cardiovascular risk-factors, including adverse lifestyle habits, and their complications in the closed rural area of Elafonisos-Island, Greece.
\end{abstract}

Methods: PERSEAS is a 5-year primary population study, with a 1-year visit elapsing period, which recruited 612 local individuals. Trained medical/paramedical personnel performed annual visits from 2012 to 2016. All participants were interviewed, and clinically evaluated.

Results: PERSEAS concluded that the Mediterranean-diet model is not faithfully adopted in the Greek rural populations. This was accompanied by physical inactivity, increased rates of obesity, hypertension, hyperlipidemia, and type 2 diabetes (35\%-50\%). Coronary heart disease (CHD, 6.1\%), and peripheral artery disease (PAD, 6.3\%) were the most prevalent cardiovascular complications compared with stroke (1.8\%), or atrial fibrillation (2\%). Older people ( $\geq 65$ years) suffered more from PAD [Odds-Ratio, 95\%C.I.:2.49 (1.03-5.98)]. Type 2 diabetes was associated with higher stroke incidence [2.61 (1.03-6.74)]. Women suffered more from stroke, CHD, or atrial-fibrillation, by almost 7-fold. Obesity was the only major risk-factor associated with all four morbidity conditions (CHD, PAD, stroke, and atrial-fibrillation).

Conclusion: Aggressive lifestyle changes and early treatment of risk-factors should be pursued to reduce the burden of cardiovascular disease in rural populations in Greece. Appropriate public health measures for improving dietary patterns, and the global trend of "Western" nutritional transition at population level should be established.

\section{Introduction}

Several epidemiological studies have demonstrated that metabolic risk-factors, such as hypertension, diabetes, and hyperlipidemia are associated with increased prevalence of cardiovascular complications $[1,2]$. Prospective studies further suggest that they are also associated with increased incidence of future cardiovascular events [3,4]. Unhealthy lifestyle, described by physical-inactivity, increased alcohol consumption, imbalanced diet, and poor sleep, increases cardiometabolic-risk, and is closely associated with a higher prevalence, and incidence of cardiovascular disease (CVD) [5,6]. Randomized controlled trials have demonstrated that medication together with healthy lifestyle measures, such as regular exercise, and diet to achieve body-weight reduction, can reduce the burden of cardiovascular morbidity $[7,8]$.

Between countries, and even among regions, there is great variability in the prevalence of cardiovascular risk-factors, and the rate of complications $[9,10]$. This has been attributed to different lifestyle, and genetic background [11]. For example, stroke incidence in Asian populations is by far higher compared with Europeans [12]; CVD incidence is higher in Northern-Europe compared with the Mediterranean-Sea [13]; and finally, higher longevity is observed in well-confined areas of one country, as was demonstrated in IkariaIsland, Greece [14].

An important problem with epidemiological studies is that the observed population may be composed of subjects with different socio-demographic, and genetic characteristics because of increased rate of inward migration. Therefore, when an epidemiological study is

${ }^{\star}$ Correspondence to: Christina Voulgari, $\mathrm{MD}, \mathrm{PhD}$, Fifth Department of Internal Medicine and Infectious Disease Unit, "Evaggelismos" Athens General Hospital, Ipsilantou 45-47, Kolonaki 10676, Athens, Greece, E-mail: c_v_24@yahoo.gr

Key words: Mediterranean diet, obesity, cardiovascular risk, lifestyle, physical exercise

Received: June 01, 2021; Accepted: June 18, 2021; Published: June 25, 2021 
prospectively planned, the observed population should be constant, and without substantial permanent outward migration. In all prospective epidemiological studies, the "Hawthorne-effect" cannot be neglected, thus the alteration of behavior by the studied subjects due to their awareness of being observed by investigators, even in the absence of interventions [15]. This phenomenon can be further increased by alternative factors, such as publicity, and diagnostic-measures, that may interfere, and change behaviors of the participants. Finally, different educational activities during the study promoting healthy lifestyle may be allowed.

In Greece, different epidemiological studies have been conducted to study the prevalence of cardiovascular risk-factors. Some of these studies were performed in open populations, like the ATTICA-study [16] in Athens. By contrast, other studies were performed in closed populations like the DIDIMA-study (Northern-Peloponnesus) [17], and the IKARIA-study [14], aiming to assess the prevalence of hypertension, and the secrets of longevity, respectively. However, all of these studies were not designed prospectively, and any reported longterm results were based on post-hoc decisions.

PERSEAS (Prospective Evaluation of vascular-Risk Surrogates: the Elafonisos-Area Study) is an epidemiological prospective study aiming to assess the physical course of cardiovascular risk-factors, including adverse lifestyle-habits, and their complications in a closed rural-area of Greece. We performed a 5-year follow-up with a 1-year visit elapsing period to promote possible beneficial changes in lifestyle-habits, and an associated change on risk-factor profile. We hoped that conclusions derived from this study, may be used for providing more targeted preventive actions against CVD in Greece.

\section{Materials and Methods}

\section{Study design}

The entire study protocol took place in the local Health Unit of Elafonisos-Island, Greece. The study was conducted under the auspices of the Greek Ministry of Health, the Municipality of Elafonisos, and the Medical Association of Lakonia, according to the guidelines by the Declaration of Helsinki. All procedures involving humans were approved by the National-Organization for Medicines which acted as an ethics/committee. The study-staff was made by trained medical/ paramedical personnel, all members of EMPAKAN, a Greek MedicalSociety for the Study of Vascular Risk-Factors. The local medicinal authority collaborated with the study investigators to provide information about the medical history of the participants. Each visit was made annually during two pre-specified weeks of each September from 2012 to 2016. The study started in 2012 (first stage) and ended in 2016 (fifth and final stage).

\section{Cohort description}

The target population consisted of all permanent inhabitants of Elafonisos-Island, aged 15years/or older, during the study-period. All participants were interviewed and underwent full clinical evaluation. The reference population (total number of the island adult inhabitants) was defined according to the 2011 census of the Greek population by the National Statistics-Agency [http://www.statistics.gr/en/2011census-pop-hous, last-accessed on 09/01/2016].

\section{Patient and public involvement}

Patients and the general public did not have direct involvement in the design of this study, recruitment, or the development of research questions, or measures. Written informed consent was obtained from all subjects. Collection and data analysis was done anonymously, but all participants had access to the results of their analyses provided to them by the local medical authority. On publication, patients will be sent a copy of the article, which would not have been possible without their participation.

\section{Materials and measurements}

\section{Dietary-score and healthy habits index}

A dietary-score was created, based on eleven questionnaireelements: whole-grain cereal, fruits/juice, vegetables/salads, legumes, fish, red-meat, poultry, full-fat dairy, olive-oil, normal-fat cheese, and alcohol consumption. Each of these ingredients' consumption levels were collapsed to two levels $(0,1)$, with sex-specific median values used as cut-off points. The sum of these values per person, corresponded to his/her dietary score. The rationale of the mapping was the following: for food categories considered to be beneficial to health value 1 was attributed for people consuming quantities above the sex-specific median. The inverse logic was applied to ingredients that are known to be non-beneficiary to health: people consuming quantities equal/ or more than the sex-specific median scored 0 . Therefore, the dietaryscore was merged into 3 categories: "Bad" (0-3)," Normal" (4-7), and" Good" (7-11) nutritional habits. Based on daily alcohol consumption value 1 was attributed to men consuming $\geq 3$ glasses/day, and for women consuming $\geq 2$ glasses/day. People consuming no alcohol at all were attributed the value 0 , irrespective of gender. Value 0 was credited to current-smokers, 1 to former-smokers, 2 to never-smokers. Regarding physical activity, we assigned 0 to $<3$ times/week, and 1 to at least 3times/week. Dietary-score, alcohol consumption, smoking, and physical activity were all incorporated into the construction of a general daily health-habits index, which ranged from 0 to 10 (unhealthy towards healthy).

\section{Other questionnaire-based variables}

All participants were asked about their educational status. The usually consumed alcoholic-beverage was registered for each participant. All participants were asked to provide the approximate number of sleepinghours/per day. Established CVD (stroke/or coronary-heart-disease, $\mathrm{CHD}$ ), and cardiovascular risk-factors (diabetes, hyperlipidemia, and hypertension) were diagnosed on the basis of patient reporting, or/and referred medical history, or/and relative medication regimen. Unknown diabetes (screen-detected) was diagnosed according to WHO-criteria, based on either a fasting-glucose $\geq 7.0 \mathrm{mmol} / \mathrm{L}$, or an $\mathrm{HbAlc} \geq 6.5 \%$. The cut-off values for diagnosing prediabetes were also based on WHO-criteria i.e., fasting-glucose $6.1-6.9 \mathrm{mmol} / \mathrm{L}$, or/ and $\mathrm{HbAlc}$ 6.0-6.4\%. Unknown hypertension was diagnosed when at least two BP measurements were $\geq 140 \mathrm{mmHg} / 90 \mathrm{mmHg}$. Additionally, unknown hyperlipidemia was considered when serum total-cholesterol was $\geq 6.2 \mathrm{mmol} / \mathrm{L}$. Peripheral artery disease (PAD) was diagnosed based either on history/or on impaired ankle-brachial-pressure-index (ABI) measures $(<0.90)$. Similarly, atrial-fibrillation (permanent/paroxysmal) diagnosis was based either on history/or on ECG-pattern performed during the visit.

\section{Measurements}

All participants underwent height/and weight measurements on site. An average of two measurements of height was obtained with a calibrated anthropometer. Body weight was determined using a digitalscale. BMI was calculated. Waist-circumference, measured at the 
Voulgari C (2021) Prospective evaluation of cardiovascular-risk surrogates via lifestyle and adherence to the Mediterranean diet: Results from PERSEAS/The Elafonisos-Area Study

midpoint between the costal-margin, and iliac-crest were also assessed. BP was measured according to the European-Society of Hypertension/ Cardiology guidelines (2013) by use of a validated electronic-device (M2 Basic Upper-Arm BP/Monitor, Omron). Hypertension was defined as systolic BP $\geq 140 \mathrm{mmHg}$, and/or diastolic BP $\geq 90 \mathrm{mmHg}$. Diagnosis of hypertension was based on two BP measurements in the sitting position. Patients with elevated BP were re-evaluated on a second visit. Capillary blood-samples were obtained to measure glucose, $\mathrm{HbA1c}$, and lipid-profile by using separate validated kits. A validated point-of-care portable analyzer was used to measure plasma lipids, and glucose-levels (CholestechLDX ${ }^{\circ}$ Laboratory Procedure, Alere) in a $40 \mu \mathrm{L}$ blood-sample, taken from a fingerstick. A second desktop point-of-care analyzer was used for measuring $\mathrm{HbAlc}$, in a blood-sample of $4 \mu \mathrm{L}$, drawn from the same fingerstick (Quo-Lab ${ }^{\text {Th }}$ A1C, EKF Diagnostics). $\mathrm{ABI}$ was measured as the ratio of the systolic $\mathrm{BP}$ at the ankle to the $\mathrm{BP}$ in the same side upper arm. Left and right $\mathrm{ABI}$ estimates were registered. Measurements were obtained by using a Doppler-device, and appropriate cuff placed over the posterior-tibial, and brachialartery. An ECG was performed in all subjects during each visit.

\section{Statistical analysis}

Apart from descriptive statistics for the 5-years of the study, inferential analysis was also performed. Due to the longitudinal nature of the study, the methods of analysis used were those of Generalized Linear Mixed-Effects Models (GLMM), Cumulative Link Mixed Models (CLMM), and Generalized Estimation Equation Models (GEE). The whole analysis was conducted using R (v.3.4.2), and R-libraries lme4 (v.1.1-10), ordinal (v.2015.6-28) and gee (v.4.13-19). GLMM and CLMM models were used appropriately, depending on the nature of the variables with participants as the grouping factor. Statistical significance level was set to $5 \%$, for all comparisons.

\section{Results}

\section{Study identity and variable changes}

The initial study population included 612 individuals $(76.5 \%$ of the island's target population), who agreed to participate, and signed an informed consent. Almost 400 individuals (65\%) provided fully completed questionnaires and underwent full clinical/laboratory assessment during the first visit. During the final (fifth) stage of the study, 352 people participated, of whom 303 were present in at least one previous stage, while 49 were first-time participants. Overall, 120 persons (17.2\%) participated in all 5 stages of the study, $88(12.6 \%)$ in four, $109(15.6 \%)$ in three, $139(19.9 \%)$ in two, while $243(34.8 \%)$ have taken part only in one stage. The age of the participants during the fifth stage was $52.7 \pm 19.6$ years (mean value \pm SD), while $35.5 \%$ of participants were $\geq 65$ years. The majority of the participants $(57.9 \%)$ had no education, while $42.1 \%$ had graduated from secondary school. BMI increased across the visits $(p=0.03)$ and measured $27.8 \mathrm{Kg} / \mathrm{m}^{2}$ in the last visit. Fruits/vegetables were consumed in a daily-basis from $40-45 \%$ of participants; fish-consumption was reported frequently (70-75\%); while olive-oil was constantly present on the table of almost all inhabitants (98.8\%). Although, there was a progressive increase in alcohol consumption $(p<0.001)$, the overall prevalence of drinkers compared with no drinkers was not different (20\%). Among alcohol beverages, wine was used more frequently $(56.4 \%)$. During the entire study-period almost $35 \%$ of participants were current-smokers, and $48 \%$ were never-smokers. Daily physical activity was performed by $8 \%$ of the participants, whereas $15 \%$ exercised 3times/weekly. The healthyhabits index was optimal/or sub-optimal (6/or 5-points respectively) in
Table 1: Association of population's characteristics with age group (Reference category: Age $<65$ years)

\begin{tabular}{|l|c|c|c|}
\hline Variable & Odds Ratio & $\mathbf{9 5 \%}$ C.I. & $\boldsymbol{p}$-value \\
\hline Age (Years) & 4.700 & $(3.67-5.72)$ & $<0.001$ \\
\hline Sleep per day (hours) & 0.570 & $(0.80-0.34)$ & $<0.001$ \\
\hline Dietary score & 1.490 & $(1.25-1.72)$ & $<0.001$ \\
\hline Height (cm) & 0.280 & $(0.14-0.41)$ & $<0.001$ \\
\hline Weight (kg) & 2.730 & $(1.00-4.47)$ & $<0.001$ \\
\hline Waist circumference (cm) & 5.020 & $(3.05-6.98)$ & $<0.001$ \\
\hline Body fat (\%) & 1.670 & $(1.65-2.68)$ & $<0.001$ \\
\hline Lean body-mass (kg) & 3.180 & $(1.17-5.20)$ & $<0.001$ \\
\hline LDL (mg/dl) & 0.720 & $(0.36-0.98)$ & $<0.001$ \\
\hline Non-HDL Cholesterol (mg/dl) & 0.640 & $(0.27-0.90)$ & 0.01 \\
\hline Fasting Glucose (mg/dl) & 11.210 & $(8.23-14.17)$ & $<0.001$ \\
\hline HbA1c (\%) & 1.310 & $(1.21-1.41)$ & $<0.001$ \\
\hline ABI (Left) & 0.010 & $(0.0-3.0)$ & 0.04 \\
\hline
\end{tabular}

Table 2: Association of population's characteristics with gender (Reference category: Males)

\begin{tabular}{|l|c|c|c|}
\hline Variable & Odds Ratio & $\mathbf{9 5 \%}$ C.I. & $\boldsymbol{p}$-value \\
\hline Age $($ Years $)$ & 6.87 & $(5.01-8.73)$ & $<0.001$ \\
\hline Dietary score & 0.31 & $(0.08-0.53)$ & 0.01 \\
\hline Weight $(\mathrm{kg})$ & 6.42 & $(4.10-8.71)$ & $<0.001$ \\
\hline Body-mass index $(\mathrm{BMI})\left(\mathrm{kg} / \mathrm{m}^{2}\right)$ & 1.53 & $(1.31-2.11)$ & $<0.001$ \\
\hline Waist circumference $(\mathrm{cm})$ & 0.83 & $(0.59-0.06)$ & $<0.001$ \\
\hline Total cholesterol $(\mathrm{mg} / \mathrm{dl})$ & 8.14 & $(3.18-13.09)$ & $<0.001$ \\
\hline HDL cholesterol $(\mathrm{mg} / \mathrm{dl})$ & 8.56 & $(6.61-10.52)$ & $<0.001$ \\
\hline Glucose $(\mathrm{mg} / \mathrm{dl})$ & 0.40 & $(0.34-0.46)$ & $<0.001$ \\
\hline HbA1c $(\%)$ & 0.11 & $(0.00-0.21)$ & 0.04 \\
\hline Ankle-Brachial Pressure Index & 0.02 & $(0.01-0.04)$ & $<0.001$ \\
\hline
\end{tabular}

almost $1 / 3$ of the participants. Overall, there was a significant increase in the percentage of hyperlipidemia at study-end $(29.6 \% v s .43 .8 \%$, $p=0.006$ ). Type 2 diabetes increased from the first to the following visits and remained constant throughout the three last years of the follow-up $(6.3 \% v s .12 .5 \%, p=0.03)$. PAD significantly increased in all study periods ( $91 \%$ vs. $96.1 \%, p=0.001)$. By study end, $52.1 \%$ of the participants were hypertensives, and $35 \%$ were obese. Of note that in the last visit, $29 \%$ of the participants had prediabetes. The overall rate of CHD, stroke, PAD, and atrial fibrillation were $6.1 \%, 1.8 \%, 6.3 \%$ and $2 \%$, respectively.

\section{Analysis by age}

As shown in Table 1, older participants had statistically significantly higher dietary-score, compared to younger persons. Waist-circumference, and body-fat percentage was higher in older participants, while height was lower. Older persons were found to get less hours of sleep per day. Elevated glucose, and HbA1c levels were both associated with age of 65 years/or more. Finally, age over 65 years was associated with lower LDL-levels, and ABI, compared to younger participants, at the time of participation.

\section{Analysis by gender}

In Table 2, statistically significant results for between gender comparisons are presented. Women demonstrated lower waistcircumference, but higher BMI, compared to men. Total-cholesterol, and HDL-cholesterol levels were higher in women. Dietary score was slightly lower in women compared to men. Additionally, glucose, HbAlc levels, and ABI, were all reported/measured lower in female participants. 
Voulgari C (2021) Prospective evaluation of cardiovascular-risk surrogates via lifestyle and adherence to the Mediterranean diet: Results from PERSEAS/The Elafonisos-Area Study

Table 3: Association of the subjects' last participation in the study with measured characteristics, for participants with two/or more visits (Reference category: First visit)

\begin{tabular}{|l|c|c|c|}
\hline Variable & Odds Ratio & $\mathbf{9 5 \%}$ CI & $\boldsymbol{p}$-value \\
\hline Weight $(\mathrm{kg})$ & 1.275 & $(1.026-1.651)$ & 0.041 \\
\hline BMI & 3.497 & $(2.264-3.551)$ & $<0.001$ \\
\hline Total -cholesterol (mg/dl) & 1.631 & $(0.614-0.648)$ & 0.004 \\
\hline LDL-cholesterol (mg/dl) & 1.709 & $(0.251-0.788)$ & $<0.001$ \\
\hline HDL-cholesterol (mg/dl) & 0.172 & $(1.431-3.913)$ & 0.015 \\
\hline HbAlc (\%) & 1.095 & $(1.029-1.162)$ & 0.005 \\
\hline ABI & 0.033 & $(0.016-0.046)$ & $<0.001$ \\
\hline Hypertension (yes) & 1.323 & $(1.161-1.845)$ & 0.003 \\
\hline Alcohol & 1.984 & $(1.35-2.952)$ & 0.001 \\
\hline Whole-grain products & 0.679 & $(0.496-0.921)$ & 0.013 \\
\hline Fruits-Juice & 0.768 & $(1.56-10.109)$ & 0.005 \\
\hline Poultry/Fish & 1.173 & $(1.028-1.755)$ & 0.031 \\
\hline Dairy products & 0.716 & $(0.525-0.967)$ & 0.03 \\
\hline Cheese & 8.579 & $(2.907-3.551)$ & $<0.001$ \\
\hline Sweets & 1.092 & $(1.033-1.223)$ & $<0.001$ \\
\hline Physical activity & 0.678 & $(0.492-0.928)$ & 0.016 \\
\hline
\end{tabular}

\section{Comparison between first and last visit}

Comparison of the measured variables' evolution between the two more distant visits, for all participants having participated at least twice, revealed that weight, and BMI had increased (Table 3). Total, and LDL-cholesterol had also increased since the participants' first visit, while HDL-cholesterol had decreased. HbAlc was found higher during the last visit, while ABI had decreased. Participants were more likely to report consuming alcohol at their last participation. Consumption of whole-grain products, fruits/juice, vegetables/salads, had decreased, whereas consumption of red-meat, and cheese had increased during the last participation. Poultry/Fish, and sweets was consumed more frequently prior to the last participation. Physical inactivity was more frequent, and hypertension was more observed at the last visit.

\section{Association between risk-factors and CVD}

Table 4 presents the association of major risk-factors with four CVD conditions recorded during the 5-years of the study: CHD, stroke, $\mathrm{PAD}$ and atrial-fibrillation. Older people ( $\geq 65$ years) suffered more from PAD, compared to their younger counterparts, after adjustment for gender, hypertension, type 2 diabetes, smoking, and hyperlipidemia. As expected, type 2 diabetes was associated with higher incidence of stroke. There was an association of gender with all CVD, except for $\mathrm{PAD}$, with females being more prone to have suffered stroke events, $\mathrm{CHD}$ or atrial-fibrillation, after adjustment for all the other risk-factors included in the model. Of importance, obesity was the only major riskfactor associated with all four CVD conditions during the 5-years study.

\section{Discussion}

\section{Main findings}

PERSEAS suggests that even in a small Eastern-Mediterranean Island like Elafonisos, the healthy diet-model based on fruits, and vegetables is not faithfully adopted. However, some of the key Mediterranean-diet elements, such as olive-oil, and fish consumption are not abandoned. Alcohol consumption was more than moderate in most of the participants, while daily physical-activity was limited. These lifestyle habits remained unchanged throughout the 5-year follow-up period, suggesting that Greek rural populations have adapted many aspects of the "Western" lifestyle, like physical inactivity, increased redmeat, and high glycemic-index products consumption. This adverse lifestyle was also accompanied by increased rates of the overweight/and obese phenotype. Moreover, the rates of hypertension, hyperlipidemia, and impaired glucose patterns (prediabetes/or type 2 diabetes) were steadily high, ranging from $35 \%$ to $50 \%$ during the different followup period visits. Participants had impaired lipid-profile, and ABI measurements from the first, and the following visits, however, this knowledge was not accompanied by prospectively trying to adapt a healthier lifestyle attitude. Cardiovascular complications demonstrated a stable representation throughout the follow-up period with CHD, and PAD being the most prevalent compared with stroke, or atrial fibrillation.

Older participants ( $\geq$ 65years) demonstrated a healthier dietary profile, and this attitude was accompanied by lower hyperlipidemia rates. However, the prevalence of type 2 diabetes was not positively affected. This may be partly attributed to the increased waistcircumference, and BMI rates observed in the elderly, thus suggesting that obesity was more prevalent. As expected, ABI was more reduced in older participants, probably due to aging. Dietary score in women was more adverse compared with men. Prevalence of hyperlipidemia, diabetes/prediabetes, and PAD were also more increased in women. Female gender was an important determinant of stroke, CHD and atrial fibrillation. Obesity was the only major metabolic risk-factor significantly, and independently associated with increased CVD risk throughout the 5 -years of study.

\section{Comparison with previous studies}

The prevalence of hypertension was more increased in PERSEAS compared with the ATTICA-study 16 performed in the early 2000 in the greater urban area of Athens, Greece ( $50 \%$ vs. $32 \%$ respectively). The same was relevant for the prevalence of hyperlipidemia (44\% in PERSEAS vs. $35 \%$ in the ATTICA). Obesity was $17 \%$ in the ATTICA cohort vs. $35 \%$ in the population of PERSEAS, while diabetes was $8 \%$ vs. $12.5 \%$ respectively. $\mathrm{CHD}$ rates were similar in the two cohorts (6\%). The adherence rate to the Mediterranean-diet was almost $30 \%$ in both the ATTICA and

Table 4: Association of risk factors with cardiovascular disease. Data are presented as Odds Ratio (95\% Confidence Interval) after adjustment for gender, age, smoking (yes), obesity (yes), hypertension (yes), Type 2 diabetes (yes), and hyperlipidemia (yes)

\begin{tabular}{|l|c|c|c|}
\hline \multicolumn{1}{|c|}{} & Stroke & Coronary heart disease & Peripheral artery disease \\
\hline Gender (Female) & $7.32(2.27-23.63)^{* * *}$ & $3.63(1.44-9.13)^{* *}$ & $1.35(0.75-2.42)$ \\
\hline Age ( $\geq 65$ years) & $1.20(0.46-3.15)$ & $0.64(0.31-1.29)$ & $2.38(1.29-4.40)^{* *}$ \\
\hline Smoking (Yes) & $1.26(0.70-2.26)$ & $1.39(0.65-2.82)$ & $0.59(0.33-1.06)$ \\
\hline Obesity (Yes) & $1.20(1.00-17.95)^{*}$ & $2.49(1.03-5.98)^{* * *}$ & $1.07(0.4-2.73)$ \\
\hline Hypertension (Yes) & $1.86(0.72-4.85)$ & $1.04(0.59-1.82)$ & $1.43(0.57-3.62)$ \\
\hline Type 2 diabetes (Yes) & $2.61(1.01-6.74)^{*}$ & $1.50(0.79-2.84)$ & $0.81(0.46-1.42)$ \\
\hline Hyperlipidemia (Yes) & $1.51(0.79-2.88)$ & $0.80(0.54-1.19)$ & $1.12(0.44-2.84)$ \\
\hline Observations (n) & 1,561 & 1,562 & $1.50(0.91-2.48)$ \\
\hline
\end{tabular}

${ }^{* * *}$ Significant at the 0.1 percent level; ${ }^{* *}$ Significant at the 1 percent level; ${ }^{*}$ Significant at the 5 percent level 
the PERSEAS studies. Although, the two populations are hardly comparable, they share similarities in the prevalence of risk-factors, and the rates of unhealthy lifestyle, thus resulting in an equal frequency of CHD. It should be acknowledged that ATTICA was conducted in a time-period during which the income in Greece was more increased, compared with the latter years in which economic-crisis is pronounced in both urban/and rural areas. In accordance with our results, recent studies indicate that during the European economic-crisis, fruit/and vegetable consumption alarmingly decreased, especially among those of lower socio-economic status, whereas trends in smoking prevalence, and physical activity were favorable $[18,19]$. These results suggest that economic-crisis has an unequal impact on different cardiovascular risk-factors among various socio-economic groups, although its overall health influence might be neutral.

The Ikaria-study [14] was a small-scale survey of the diet, and lifestyle of elderly Greeks (>80years) on Ikaria-Island. The main findings revealed a lower prevalence of cardiovascular risk-factors in the elderly cohort, attributed to a higher adherence to the Mediterraneandiet, habitual physical activity, noon-siesta, and engagement in social activities. The prospective results of the Ikaria-study are lacking in PERSEAS. Although, the elderly cohort of PERSEAS was more attached to a healthier lifestyle compared with the younger people, there was a high prevalence of cardiovascular risk-factors in both age-related strata (>65years $v s .<65$ years). Taken together the elderly people in both Ikaria and Elafonisos-Island demonstrated an overall healthy lifestyle, but this attitude in Elafonisos, at variance with Ikaria, was not associated with decreased rates of CVD morbidity.

\section{Conclusions}

The present study demonstrated a marginal adherence to the Mediterranean-diet, and a high risk-factor prevalence in the rural population of Greece. Among cardiovascular risk-factors the prevalence of hypertension was almost 50\%, type 2 diabetes $13 \%$, hyperlipidemia $45 \%$, and obesity $35 \%$. High rates of overweight phenotype, and prediabetic state were also observed (30\%). These adverse lifestyle, and risk-factor profiles increased throughout the 5-year follow-up period. In the rural area of Elafonisos, cardiovascular complications were not different compared with those of an urban area of Greece, and were strongly related to female gender, and obesity status. Our findings confirm that aggressive lifestyle changes, and early treatment of riskfactors should be pursued to reduce the burden of CVD in Greece. Strategies against the obesity epidemic might be a crucial intervention to reduce heighten cardiovascular risk. Therefore, it is important to establish appropriate public health measures for improving dietary patterns, and the global trend of "Western" nutritional transition at the population level.

\section{Funding}

This study was funded by Mylan SA. The funders had no involvement in study design, collection, management, analysis and data interpretation, writing of the report and the decision to submit the report for publication.

\section{Author Contributions}

(I) Conception and design: I. Kyriazis, I. Ioannidis, Ch. Dimosthenopoulos, E. Hatziagelaki (II) Administrative support: All authors, (III) Provision of study material or patients: I. Kyriazis, I. Ioannidis, Ch. Dimosthenopoulos, E. Hatziagelaki (IV) Collection and assembly of data: I. Kyriazis, I. Ioannidis, Ch. Dimosthenopoulos, E. Hatziagelaki, (V) Data analysis and interpretation: C. Voulgari, I. Kyriazis, (VI) Manuscript writing: C. Voulgari, (VII).

\section{References}

1. Palomo L, Félix-Redondo FJ, Lozano-Mera L, Pérez-Castán JF, Fernández-Berges D, et al. (2014) Cardiovascular risk factors, lifestyle, and social determinants: a crosssectional population study. Br J Gen Pract 64: e627-633. [Crossref]

2. Zhang FL, Guo ZN, Wu YH, Liu HY, Luo Y, et al. (2017) Prevalence of stroke and associated risk factors: a population based cross sectional study from northeast China. BMJ Open 7: e015758. [Crossref]

3. Tsao CW, Vasan RS (2015) Cohort Profile: The Framingham Heart Study (FHS) overview of milestones in cardiovascular epidemiology. Int J Epidemiol 44: 18001813. [Crossref]

4. Guo F, Garvey T (2016) Trends in cardiovascular health metrics in obese adults: National Health and Nutrition Examination Survey (NHANES), 1998-2014. J Am Coll Cardiol 5: e003619. [Crossref]

5. Hulsegge G, Looman M, Smit HA, Daviglus ML, van der Schouw YT, et al. (2016) Lifestyle Changes in Young Adulthood and Middle Age and Risk of Cardiovascular Disease and All-Cause Mortality: The Doetinchem Cohort Study. J Am Heart Assoc 5: e002432. [Crossref]

6. Zhang QL, Zhao LG, Zhang W, Li HL, Gao J, et al. (2017) Combined Impact of Known Lifestyle Factors on Total and Cause-Specific Mortality among Chinese Men: A Prospective Cohort Study. Sci Rep 7: 5293. [Crossref]

7. Thomopoulos C, Parati G, Zanchetti A (2014) Effects of blood pressure lowering on outcome incidence in hypertension. 1. Overview, meta-analyses, and meta-regression analyses of randomized trials. J Hypertens 32: 2285-2295. [Crossref]

8. Diabetes Prevention Program Research Group (2015) Long-term effects of lifestyle intervention or metformin on diabetes development and microvascular complications over 15-year follow-up: the Diabetes Prevention Program Outcomes Study. Lancet Diabetes Endocrinol 3: 866-875. [Crossref]

9. Hirte L, Nolte E, Mossialos E, McKee M (2008) The changing regional pattern of ischaemic heart disease mortality in southern Europe: still healthy but uneven progress. $J$ Epidemiol Community Health 62: e4. [Crossref]

10. Benjamin EJ, Virani SS, Callaway CW, Chamberlain AM, Chang AR, et al. (2018) Heart Disease and Stroke Statistics-2018 Update: A Report From the American Heart Association. Circulation 137: e67-e492. [Crossref]

11. Volgman AS, Palaniappan LS, Aggarwal NT, Gupta M, Khandelwal A, et al. (2018) Atherosclerotic Cardiovascular Disease in South Asians in the United States: Epidemiology, Risk Factors, and Treatments: A Scientific Statement From the American Heart Association. Circulation 138: e1-e34. [Crossref]

12. Thomopoulos C, Katsimagklis G, Makris T (2018) Morning hypertension in Asian populations. J Clin Hypertens (Greenwich) 20: 45-46. [Crossref]

13. Panagiotakos DB, Fitzgerald AP, Pitsavos C, Pipilis A, Graham I, et al. (2007) Statistica modelling of 10-year fatal cardiovascular disease risk in Greece: the HellenicSCORE (a calibration of the ESC SCORE project). Hellenic J Cardiol 48: 55-63. [Crossref]

14. Panagiotakos DB, Chrysohoou C, Siasos G, Zisimos K, Skoumas J, et al. (2011) Sociodemographic and lifestyle statistics of oldest old people ( $>80$ years) living in ikaria island: the ikaria study. Cardiol Res Pract 2011: 679187. [Crossref]

15. Sedgwick P, Greenwood N (2015) Understanding the Hawthorne effect. BMJ 351: h4672. [Crossref]

16. Pitsavos C, Panagiotakos DB, Chrysohoou C, Stefanadis C (2003) Epidemiology of cardiovascular risk factors in Greece: aims, design and baseline characteristics of the ATTICA study. BMC Public Health 3: 32. [Crossref]

17. Stergiou GS, Baibas NM, Kalogeropoulos PG (2007) Cardiovascular risk prediction based on home blood pressure measurement: the Didima study. J Hypertens 25: 15901596. [Crossref]

18. Monsivais P, Aggarwal A, Drewnowski A (2012) Are socio-economic disparities in diet quality explained by diet cost? J Epidemiol Community Health 66: 530-535. [Crossref]

19. Filippidis FT, Schoretsaniti S, Dimitrakaki C, Vardavas CI, Behrakis P, et al. (2014) Trends in cardiovascular risk factors in Greece before and during the financial crisis: the impact of social disparities. Eur J Public Health 24: 974-979. [Crossref]

Copyright: (C2021 Voulgari C. This is an open-access article distributed under the terms of the Creative Commons Attribution License, which permits unrestricted use, distribution, and reproduction in any medium, provided the original author and source are credited. 